\title{
Thermal ablation versus cryotherapy or loop excision to treat women positive for cervical precancer on visual inspection with acetic acid test: pilot phase of a randomised controlled trial
}

Leeya F Pinder, Groesbeck P Parham, Partha Basu, Richard Muwonge, Eric Lucas, Namakau Nyambe, Catherine Sauvaget, Mulindi H Mwanahamuntu, Rengaswamy Sankaranarayanan, Walter Prendiville

\section{Summary}

Background Cryotherapy is standard practice for treating patients with cervical precancer in see-and-treat programmes in low-income and middle-income countries (LMICs). Because of logistical difficulties with cryotherapy (eg, the necessity, costs, and supply chain difficulties of refrigerant gas; equipment failure; and treatment duration $>10 \mathrm{~min}$ ), a battery-operated thermal ablator that is lightweight and portable has been developed. We aimed to compare thermal ablation using the new device with cryotherapy.

Methods We report the pilot phase of a randomised controlled trial in routine screen-and-treat clinics providing cervical screening using visual inspection with acetic acid (VIA) in Lusaka, Zambia. We recruited non-pregnant women, aged 25 years or older, who were eligible for ablative therapy. We randomly assigned participants (1:1:1) to thermal ablation, cryotherapy, or large loop excision of the transformation zone (LLETZ), using computer-generated allocation. The randomisation was concealed but the nurses providing treatment and the participants were unmasked. Thermal ablation was achieved using the Liger thermal ablator (using 1-5 overlapping applications of the probe heated to $100^{\circ} \mathrm{C}$, each application lasting for $40 \mathrm{~s}$ ), cryotherapy was carried out using the double-freeze technique (freeze for $3 \mathrm{~min}$, thaw for $5 \mathrm{~min}$, and freeze again for $3 \mathrm{~min}$ ), and LLETZ (using a large loop driven by an electrosurgical unit to excise the transformation zone) was done under local anaesthesia. The primary endpoint was treatment success, defined as either human papillomavirus (HPV) type-specific clearance among participants who were positive for the same HPV type at baseline, or a negative VIA test at 6-month follow-up, if the baseline HPV test was negative. Per protocol analyses were done. Enrolment for the full trial is ongoing. Here, we present findings from a prespecified pilot phase of the full trial. The final analysis of the full trial will assess non-inferiority of the groups for the primary efficacy endpoint. The study is registered with ClinicalTrials.gov, number NCT02956239.

Findings Between Aug 2, 2017, and Jan 15, 2019, 750 participants were randomly assigned (250 per group). 206 (84\%) participants in the cryotherapy group, $197(81 \%)$ in the thermal ablation group, and $204(84 \%)$ in the LLETZ group attended the 6-month follow-up examination. Treatment success was reported in $120(60 \%)$ of 200 participants in the cryotherapy group, 123 (64\%) of 192 in the thermal ablation group, and 134 (67\%) of 199 in the LLETZ group (p=0 • 31). Few participants complained of moderate to severe pain in any group immediately after the procedure (six [2\%] of 250 in the cryotherapy group, four [2\%] of 250 in the thermal ablation group, and five [2\%] of 250 in the LLETZ group) and 2 weeks after the procedure (one $[<1 \%]$ of 241 in the cryotherapy group, none of 242 in the thermal ablation group, and two [<1\%] of 237 in the LLETZ group). None of the participants reported any complication requiring medical consultation or admission to hospital.

Interpretation Results from this pilot study preliminarily suggest that thermal ablation has similar treatment success to cryotherapy, without the practical disadvantages of providing cryotherapy in an LMIC. However, the study was not powered to establish the similarity between the techniques, and results from the ongoing randomised controlled trial are need to confirm these results.

Funding US National Institutes of Health.

\section{Introduction}

Systematic high coverage and quality-assured population screening, with treatment of precursors to cervical cancer, is highly effective i $\mathrm{n} p$ reventing the $\mathrm{d}$ isease, which is not surprising given that the conditions for an ideal screening test ${ }^{1}$ apply very precisely to cervical cancer. The disease has a long precancerous phase, effective easy screening tests are available, treatment of precursors is highly effective, and the disease is common enough to justify the expense of population screening,
Lancet Oncol 2020; 21: 175-84

Published Online

November 13, 2019

https://doi.org/10.1016/

S1470-2045(19)30635-7

See Comment page 19

Department of Obstetrics and Gynecology, University of Washington, Seattle, WA, USA (LF Pinder MD); Department of Obstetrics and Gynecology, Women and Newborn Hospital (MH Mwanahamuntu MD), Obstetrics and Gynecology, (LF Pinder, Prof G P Parham MD), University of Zambia, Lusaka, Zambia); Department of Obstetrics and Gynecology, University of North Carolina, Chapel Hill, NC, USA

(Prof G P Parham); Screening Group, Early Detection and Prevention Section, International Agency for Research on Cancer, WHO Lyon, France (P Basu MD, R Muwonge PhD, E Lucas MSc, C Sauvaget MD

W Prendiville MD); UNC Global Project-Zambia, Lusaka, Zambia (N Nyambe MA); and Research Triangle Institute, International-India,

Commercial Tower, Pullman Hotel Aerocity, New Delhi, India (R Sankaranarayanan MD) Correspondence to:

Dr Partha Basu, Screening Group, Early Detection and Prevention Section, International Agency for Research on Cancer, WHO, Lyon 69372, France basup@iarc.fr 
Research in context

\section{Evidence before this study}

The see-and-treat approach, by reducing the number of clinic visits, improves treatment compliance in a cervical cancer screening programme in resource-limited settings. Screening cervical cancer by visual inspection with acetic acid (VIA) followed by immediate cryotherapy is the most commonly used approach in low-income and middle-income countries (LMICs). Thermal ablation is an alternative ablative procedure. WHO has endorsed cryotherapy as the standard method of treatment for patients with cervical pre-cancer in LMICs for more than a decade. Although cold coagulation (now known as thermal ablation) has been in use for many years in the UK and elsewhere, and despite evidence from large and long-term follow-up studies in Scotland, the method has not been accepted in LMICs, perhaps largely because of the WHO endorsement of cryotherapy. We searched PubMed with no language or date restrictions using the keywords "cervical intraepithelial neoplasia OR CIN OR cervical precancerous lesions" and "ablative treatment", and "LMICs" on Sept 7, 2018, for articles describing this ablative procedure in low-resource settings. We also checked the reference lists of the selected articles. A meta-analysis of the available published evidence revealed similar effectiveness of cryotherapy and thermal ablation. We found only one small randomised controlled trial in the studies included.

even in low-income and middle-income countries (LMICs). ${ }^{2}$ Large loop excision of the transformation zone (LLETZ), ${ }^{3}$ also known as loop electrosurgical excision procedure (LEEP), has become the standard treatment in most high-income countries. Ablative techniques are simpler, safer, and less technically demanding than LLETZ. Available ablative methods are cryotherapy and thermal ablation. The techniques have been described in detail elsewhere. ${ }^{4}$ Thermal ablation was previously known as cold coagulation to distinguish it from radical diathermy, which reaches temperatures of approximately $300^{\circ} \mathrm{C}$. $^{5}$ Thermal ablation functions by heating the epithelium at the transformation zone, albeit to $100^{\circ} \mathrm{C}$.

Screening by visual inspection with acetic acid (VIA), ${ }^{6}$ followed by immediate treatment of VIA-positive women (screen-and-treat approach) can reduce the number of clinic visits by women and greatly improve treatment compliance. ${ }^{7}$ Cryotherapy was previously recommended by WHO as the ablative method of choice for screenand-treat programmes in LMICs. ${ }^{8}$ The method has the advantage of not requiring electricity, being simple to use, and being effective. However, the costs and difficulties in ensuring uninterrupted supply of $\mathrm{CO}_{2}$ or $\mathrm{N}_{2} \mathrm{O}$ refrigerant gas, the long treatment duration (11 $\mathrm{min}$ ), and difficulties with equipment failure have led to the frustration of treatment providers with the method. ${ }^{9,10}$ Thermal ablation is an alternative ablation therapy to cryotherapy. Similar effectiveness between the

\section{Added value of this study}

The results of the pilot phase of our study revealed a similarity in efficacy between thermal ablation and cryotherapy, although the pilot study was not adequately powered because of the small sample size. We found no difference in complication or discomfort levels between the study groups. The excisional group revealed that only $25 \%$ of participants who were deemed to be screen positive and eligible for ablation had high-grade squamous lesions. The study adds valuable evidence for similar efficacy and safety of the two ablative techniques. The high rate of over-treatment in a screen-and-treat setting has been quantified.

\section{Implications of all the available evidence}

The study will continue until sufficient power has been achieved to establish equivalent efficacy between thermal ablation and cryotherapy. The results of this pilot study suggest that thermal ablation as a method of treating cervical precancers is as safe as cryotherapy and is highly acceptable to patients and providers. If the early results of the pilot study regarding the similarity of treatment efficacy between thermal ablation and cryotherapy are supported by our ongoing randomised controlled trial, thermal ablation without the practical disadvantages of cryotherapy will be the ablative treatment of choice in an LMIC setting.

two methods has been shown in a pooled analysis of published observational studies. ${ }^{11,12}$ The method has a much faster treatment duration (20-40 s) and requires no gas supply. Like cryotherapy, thermal ablation is simple to use and could be given by almost any healthcare provider.

Consequently, the search for a simpler, affordable, and mobile ablative treatment modality to incorporate into see-and-treat regimes in LMICs has led to the development of a cordless, lightweight, and batteryoperated thermal ablator. The International Agency for Research on Cancer (IARC) and the University of North Carolina (NC, USA) collaborated with Liger Medical (UT, USA) to assess the new device. Here, we report the pilot phase outcomes of a three-group randomised controlled trial of thermal ablation using the new portable device compared with cryotherapy and LLETZ in the context of a VIA-based screen-and-treat programme in Lusaka, Zambia. We aimed to compare the success of the three treatment methods. We also aimed to estimate the proportion of over-treatment in a VIA screen-and-treat programme on the basis of the histopathology results after LLETZ in the LLETZ group.

\section{Methods \\ Study design and participants}

This prospective, unblinded, randomised trial was done in a primary health clinic participating in the routine 
screen-and-treat programme in Lusaka, Zambia, where VIA is done by trained nurses to screen women aged between 25 and 49 years. Like many other LMICs, the access to quality assured cytology is poor in Lusaka and we, therefore, decided to adhere to the national protocol of VIA-based screening followed by treatment of screenpositive women.

Here, we present findings from a prespecified pilot phase of the full trial. This pilot phase was done on request of the funder, a condition set before further funding for the full trial could be awarded.

All women attending the study VIA screening clinic were counselled about the trial by a research nurse before going to the clinic room. VIA was performed done as described by the IARC manual on VIA, ${ }^{13}$ and the VIA outcomes were categorised as negative, positive, and suspected cancer. ${ }^{13}$ The examining nurses assessed the eligibility of VIA-positive women for ablative treatment. These eligibility criteria were that the transformation zone be a type 1 (completely ectocervical), not involving more than $75 \%$ of the ectocervix, not extending to the vagina, and with no suspicion of cancer., ${ }^{4,14}$ Women who were eligible for ablative treatment by the clinic nurse were invited to participate in the trial. Eligible women who agreed to take part in the study then gave written, informed consent. Exclusion criteria were any reason whereby informed consent was not freely given, not eligible for ablative treatment, size of the lesion was such that it could not be covered by the largest cryotherapy probe, pregnancy, previous treatment to the cervix for any reason, and any genital tract cancer.

As per routine practice in Zambia, all women undergoing VIA underwent HIV testing, unless a test result from within the past 6 months was available. Recently diagnosed HIV positivity required initiation of antiretroviral therapy before cervical cancer screening.

This study was approved by the research ethics committee at IARC, the University of North Carolina, the University of Zambia, and the National Health Research Agency of Zambia. The full trial protocol can be provided on request.

\section{Randomisation and masking}

Eligible participants were randomly assigned (1:1:1) to receive either thermal ablation, cryotherapy, or LLETZ. All treatment was given by one of four study nurses at the clinic. A request for allocation was obtained by the study nurse after checking the inclusion and exclusion criteria. Concealed allocation to a study group was done using computer-generated sealed envelopes at IARC, which were accessed by the study coordinator in the clinic. Study group allocation was conveyed to the nurse immediately before treating eligible participants. Once a treatment group had been allocated, the participant received a unique identifier number. Neither the treating nurse, nor the participant, were masked to the treatment allocation.

\section{Procedures}

The nurse collected a cervical sample before VIA using a Cervex-brush (Rovers Medical Devices [Oss, the Netherlands]) in Preservcyt medium (Hologic [Marlborough, MA, USA]) for human papillomavirus (HPV) DNA testing. If a woman was randomly assigned, her sample was sent to the University Teaching Hospital, Lusaka, laboratory for the detection of DNA of any of the 14 high-risk HPV types (with typespecific information) using the Xpert HPV test (Cepheid [Sunnyvale, CA, USA]). The HPV genotype information was obtained in separate channels for HPV 16; HPV 18 and 45; HPV 31, 33, 35, 52, and 58; HPV 51 and 59; and HPV 39, 56, 66, and 68. The test results were obtained after randomisation and treatment and did not alter treatment allocation or the management of eligible VIA-positive women.

Thermal ablation was done using the Liger thermal ablator and as described in the IARC colposcopy manual. ${ }^{15}$ The portable battery-driven thermal ablator was developed by Liger Medical (Lehi, UT, USA) during 2016 and 2017 and bench tested in 2017. US Food and Drug Administration clearance was obtained in 2017, as was the European CE mark. The device is powered by a small removable 12-volt battery that is incorporated into the handle, which can be recharged over 2-3 h and holds enough charge to complete at least 20 treatment procedures. The thermal ablator probe was heated to $100^{\circ} \mathrm{C}$ and applied over the transformation zone of the cervix for $40 \mathrm{~s}$. Up to five overlapping applications, each lasting for $40 \mathrm{~s}$ were used to treat a large transformation zone.

Cryotherapy was carried out using the double-freeze technique (freeze for $3 \mathrm{~min}$, thaw for $5 \mathrm{~min}$, and freeze again for $3 \mathrm{~min}$ ) as per routine practice in screen-andtreat programmes in Zambia. ${ }^{4}$ LLETZ was done under local anaesthesia, as described by Prendiville and colleagues. ${ }^{3}$ Briefly, a type 1 excision of the transformation zone was done using a large yet shallow metallic loop, following local infiltration with $1 \%$ lignocaine. No anaesthesia was used for either thermal ablation or cryotherapy. Any treatment side-effects during and immediately after treatment were recorded by the nurse providing the treatment. Safety was assessed as any major complications leading to hospitalisation, disability, or death.

The treating nurse counselled each participant after the procedure about possible side-effects and complications of treatment and advised participants to report to the clinic or call the study coordinator for advice. Abstinence from sexual intercourse for 6 weeks and avoidance from douching or any vaginal medications was advised. Neither analgesics nor antibiotics were prescribed. Every participant was invited to attend a follow-up clinic appointment at 6 months. Before leaving the clinic, participants were interviewed by the project coordinator to document 


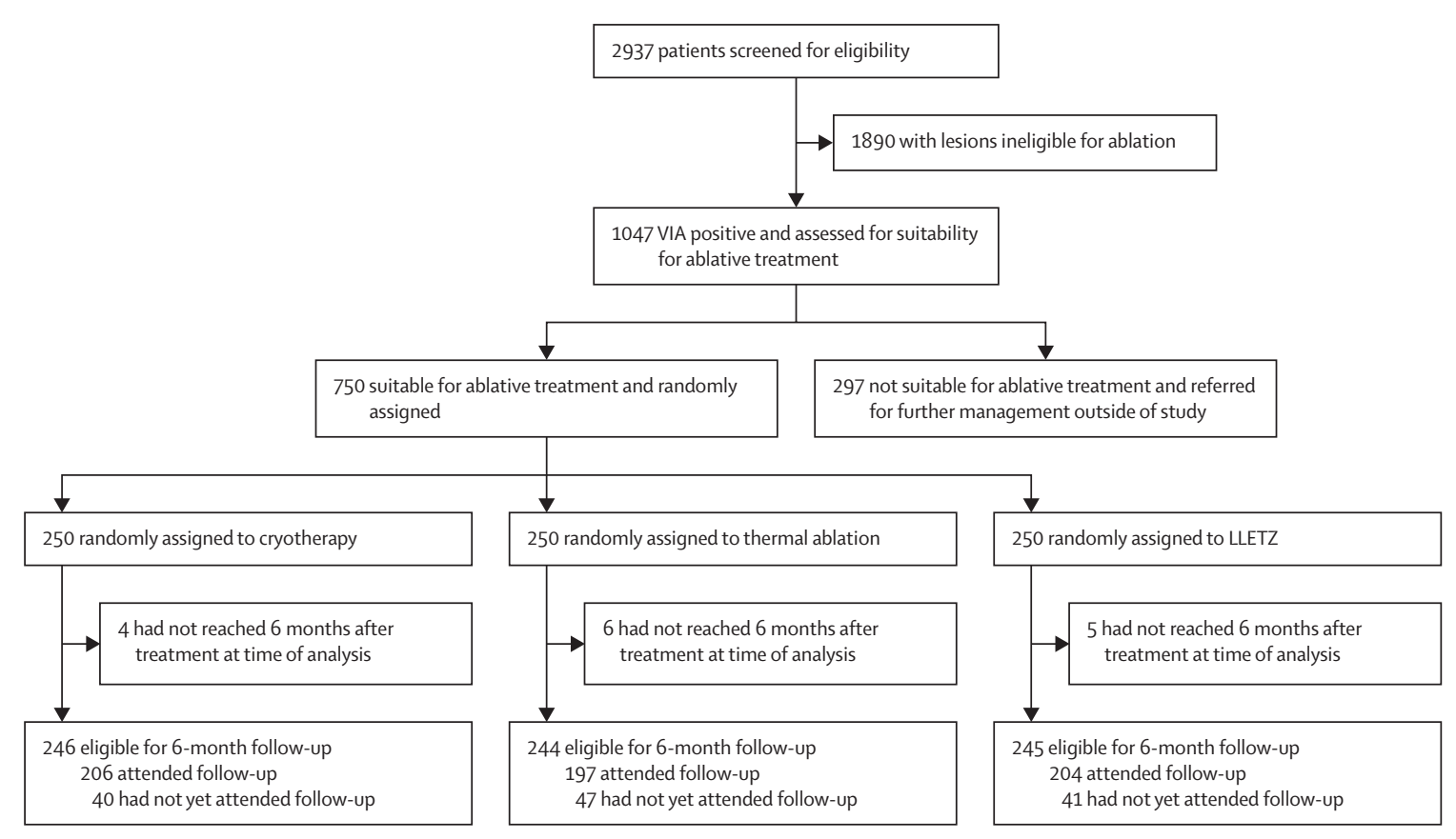

Figure: Trial profile

VIA=visual inspection with acetic acid. LLETZ=large loop excision of the transformation zone.

their perception of pain and discomfort during and immediately after treatment and also their satisfaction with the overall experience. Pain and satisfaction were assessed using a visual rating scale ranging from 1 (no pain at all or was highly satisfied) to 9 (pain was so severe that the participant wanted the procedure to be stopped or was not at all satisfied).

The study coordinator phoned each participant 2 weeks after treatment to check whether she had any complications, had visited a clinician, or had been admitted to hospital during the intervening period. The level of pain and discomfort and the degree of satisfaction with the overall experience at that timepoint was again recorded.

At the 6-month follow-up visit, each participant was asked about any complications, medical consultations, or hospitalisations. Participants were examined by a study nurse, who first collected a cervical sample for Xpert HPV testing and did VIA screening. VIA-positive participants were immediately referred for further assessment and appropriate management as per the local protocol. Participants who were negative on VIA, but positive for HPV, were advised to attend a repeat follow-up visit at 12 months.

\section{Outcomes}

The primary outcome was success of treatment at 6 months, which was defined as either HPV typespecific clearance at 6 months among participants positive for the same HPV type at baseline, or negative VIA test at follow-up, if the baseline HPV test was negative. The secondary outcomes were safety and acceptability of the three treatment methods. The proportion of participants undergoing LLETZ and having cervical intraepithelial neoplasias (CIN) on histopathology was also assessed as a secondary outcome, which helped us to assess the degree of overtreatment in screen-and-treat settings.

\section{Statistical analysis}

The sample size of 250 participants per group that was used for the assessment of the preliminary safety and efficacy was empirically decided. Recruitment for the full trial is ongoing and is expected to complete recruitment and follow-up within 2 years. A data and safety monitoring board continues to oversee the project.

The primary outcome was analysed per protocol. Treatment success, side-effects, complications, and acceptability presented as proportions and were compared between the three treatment modalities using a two-tailed Fisher's exact test. The interval between treatment and follow-up was compared using the Kruskal-Wallis equalityof-populations rank test. Statistical significance was set as $\mathrm{p}<0 \cdot 05$. Treated participants who had not yet reported for their 6-month follow-up (including those who were not yet eligible or those who were eligible, but did not attend a follow-up visit) were deemed not assessable in the analysis of the primary endpoint in the pilot phase. Statistical analyses were done using STATA (version 14.0) and $\mathrm{R}$ (version 3.6.0).

This study is registered with ClinicalTrials.gov, numberNCT02956239. 


\begin{tabular}{|c|c|c|c|}
\hline & $\begin{array}{l}\text { Cryotherapy } \\
\text { group }(n=250)\end{array}$ & $\begin{array}{l}\text { Thermal ablation } \\
\text { group }(n=250)\end{array}$ & $\begin{array}{l}\text { LLETZ group } \\
(\mathrm{n}=250)\end{array}$ \\
\hline \multicolumn{4}{|c|}{ Age range, years } \\
\hline $25-29$ & $92(37 \%)$ & $89(356 \%)$ & $84(34 \%)$ \\
\hline $30-34$ & $54(22 \%)$ & $65(26 \%)$ & $64(26 \%)$ \\
\hline 35-39 & $44(18 \%)$ & $37(15 \%)$ & 47 (19\%) \\
\hline $40-44$ & $35(14 \%)$ & $31(12 \%)$ & $37(15 \%)$ \\
\hline $45-49$ & $15(6 \%)$ & $17(7 \%)$ & $11(4 \%)$ \\
\hline $50-54$ & $9(4 \%)$ & $8(3 \%)$ & $6(2 \%)$ \\
\hline $55-60$ & $1(<1 \%)$ & $3(1 \%)$ & $1(<1 \%)$ \\
\hline \multicolumn{4}{|l|}{ Education } \\
\hline None & $9(4 \%)$ & $7(3 \%)$ & $10(4 \%)$ \\
\hline Primary & $88(35 \%)$ & $75(30 \%)$ & $74(30 \%)$ \\
\hline Secondary & $101(40 \%)$ & $128(51 \%)$ & $123(49 \%)$ \\
\hline $\begin{array}{l}\text { College or } \\
\text { university }\end{array}$ & $50(20 \%)$ & $40(16 \%)$ & $41(16 \%)$ \\
\hline Unknown & $2(<1 \%)$ & 0 & $2(<1 \%)$ \\
\hline \multicolumn{4}{|l|}{ Occupation } \\
\hline Housewife & $62(25 \%)$ & $68(27 \%)$ & $57(23 \%)$ \\
\hline Manual & $26(10 \%)$ & $23(9 \%)$ & $29(12 \%)$ \\
\hline Professional & $43(17 \%)$ & $44(18 \%)$ & $37(15 \%)$ \\
\hline Business & 92 (37\%) & $81(32 \%)$ & $86(34 \%)$ \\
\hline Other & $16(6 \%)$ & $29(12 \%)$ & $31(12 \%)$ \\
\hline Unknown & $11(4 \%)$ & $5(2 \%)$ & $10(4 \%)$ \\
\hline \multicolumn{4}{|c|}{ Marital status } \\
\hline Unmarried & $34(14 \%)$ & $32(13 \%)$ & $35(14 \%)$ \\
\hline $\begin{array}{l}\text { Married or } \\
\text { cohabiting }\end{array}$ & $168(67 \%)$ & $171(68 \%)$ & $157(63 \%)$ \\
\hline Widowed & 19 (8\%) & $17(7 \%)$ & $24(10 \%)$ \\
\hline Separated & $28(11 \%)$ & $30(12 \%)$ & $34(14 \%)$ \\
\hline Unknown & $1(<1 \%)$ & 0 & 0 \\
\hline \multicolumn{4}{|c|}{ Residence area } \\
\hline Urban & $146(58 \%)$ & $152(61 \%)$ & 149 (60\%) \\
\hline Semiurban & $92(37 \%)$ & $90(36 \%)$ & $94(38 \%)$ \\
\hline Rural & $11(4 \%)$ & $7(3 \%)$ & $7(3 \%)$ \\
\hline Unknown & $1(<1 \%)$ & $1(<1 \%)$ & 0 \\
\hline \multicolumn{4}{|c|}{ Total pregnancies } \\
\hline None & $21(8 \%)$ & $14(6 \%)$ & $20(8 \%)$ \\
\hline $1-2$ & $74(30 \%)$ & 88 (35\%) & $102(41 \%)$ \\
\hline $3-4$ & 93 (37\%) & $90(36 \%)$ & 79 (32\%) \\
\hline$\geq 5$ & $62(25 \%)$ & $58(23 \%)$ & $49(20 \%)$ \\
\hline
\end{tabular}

\begin{tabular}{|c|c|c|c|}
\hline & $\begin{array}{l}\text { Cryotherapy } \\
\text { group }(n=250)\end{array}$ & $\begin{array}{l}\text { Thermal ablation } \\
\text { group }(n=250)\end{array}$ & $\begin{array}{l}\text { LLETZ group } \\
(n=250)\end{array}$ \\
\hline \multicolumn{4}{|c|}{ (Continued from previous column) } \\
\hline \multicolumn{4}{|l|}{ Total livebirths } \\
\hline None & $28(11 \%)$ & $22(9 \%)$ & $29(12 \%)$ \\
\hline $1-2$ & $97(39 \%)$ & $109(44 \%)$ & $120(48 \%)$ \\
\hline $3-4$ & $87(35 \%)$ & $85(34 \%)$ & $71(28 \%)$ \\
\hline$\geq 5$ & $38(15 \%)$ & $34(14 \%)$ & $30(12 \%)$ \\
\hline \multicolumn{4}{|c|}{ Last menstruation } \\
\hline$\leq 30$ days & $217(87 \%)$ & $213(85 \%)$ & $215(86 \%)$ \\
\hline $\begin{array}{l}>30 \text { days to } \\
<12 \text { months }\end{array}$ & $18(7 \%)$ & $21(8 \%)$ & $22(9 \%)$ \\
\hline$\geq 12$ months & $2(<1 \%)$ & $6(2 \%)$ & $3(1 \%)$ \\
\hline Unknown & $13(5 \%)$ & $10(4 \%)$ & $10(4 \%)$ \\
\hline \multicolumn{4}{|c|}{ Size of the acetowhite area } \\
\hline$<50 \%$ of TZ & $221(88 \%)$ & $223(89 \%)$ & $210(84 \%)$ \\
\hline$>50 \%$ of TZ & $29(12 \%)$ & $27(11 \%)$ & $40(16 \%)$ \\
\hline \multicolumn{4}{|c|}{ Baseline HIV status } \\
\hline Negative & $108(43 \%)$ & $119(48 \%)$ & $110(44 \%)$ \\
\hline Positive & $134(54 \%)$ & $123(49 \%)$ & $135(54 \%)$ \\
\hline Unknown & $8(3 \%)$ & $8(3 \%)$ & $5(2 \%)$ \\
\hline \multicolumn{4}{|c|}{ HIV positive on ART } \\
\hline Yes & $129 / 134(96 \%)$ & $117 / 123(95 \%)$ & $130 / 135(96 \%)$ \\
\hline No & $4 / 134(3 \%)$ & $5 / 123(4 \%)$ & $1 / 135(<1 \%)$ \\
\hline Unknown & $1 / 134(<1 \%)$ & $1 / 123(<1 \%)$ & $4 / 135(3 \%)$ \\
\hline \multicolumn{4}{|c|}{ HPV testing results } \\
\hline Negative & $100(40 \%)$ & $113(45 \%)$ & $105(42 \%)$ \\
\hline Positive & $150(60 \%)$ & $136(55 \%)$ & $145(58 \%)$ \\
\hline Unknown & 0 & $1(<1 \%)$ & 0 \\
\hline \multicolumn{4}{|l|}{ HPV type* } \\
\hline HPV 16 & $38(15 \%)$ & $41(17 \%)$ & $51(20 \%)$ \\
\hline HPV 18 or 45 & $24(10 \%)$ & $21(8 \%)$ & $21(8 \%)$ \\
\hline $\begin{array}{l}\text { HPV 31, 33, 35, } \\
52,58\end{array}$ & $99(40 \%)$ & $94(38 \%)$ & $101(40 \%)$ \\
\hline HPV 51 or 59 & $20(8 \%)$ & $11(4 \%)$ & $12(5 \%)$ \\
\hline $\begin{array}{l}\text { HPV } 39,56,66 \text {, } \\
\text { or } 68\end{array}$ & $60(24 \%)$ & $45(18 \%)$ & $54(22 \%)$ \\
\hline \multicolumn{4}{|c|}{$\begin{array}{l}\text { Data are } n(\%) \text { or } n / N(\%) \text {. LLETZ=large loop excision of the transformation zone. } \\
\text { ART=antiretroviral therapy. HPV=human papillomavirus. *Some patients had } \\
\text { combinations of multiple HPV genotypes. }\end{array}$} \\
\hline
\end{tabular}

participants were recruited. Participants were randomly assigned to thermal ablation $(\mathrm{n}=250)$, cryotherapy $(\mathrm{n}=250)$, or LLETZ $(\mathrm{n}=250)$. Table 1 provides details of the baseline sociodemographic, reproductive, and clinical characteristics between the three treatment groups.

In the cryotherapy group, 246 (98\%) participants completed 6 months of treatment and were eligible for 6-month follow-up, of whom 206 (84\%) attended the 6-month follow-up examination. In the thermal ablation group, $244(98 \%)$ participants were eligible for 6-month follow-up and 197 (81\%) attended the 6-month follow-up examination, and in the LLETZ group, $245(98 \%)$ were eligible for 6-month follow-up and 204 (84\%) attended the 6-month follow-up examination. The overall median 
Cryotherapy Thermalablation LLETZ group Fisher's exact group group pvalue

\begin{tabular}{|c|c|c|c|c|}
\hline \multicolumn{5}{|l|}{ Overall } \\
\hline Participants followed up* & 200 & 192 & 199 & .. \\
\hline $\begin{array}{l}\text { Participants with no evidence of } \\
\text { disease }\end{array}$ & $120(60 \%)$ & $123(64 \%)$ & $134(67 \%)$ & 0.52 \\
\hline \multicolumn{5}{|l|}{ HPV positive at baseline } \\
\hline High-risk participants followed up & 121 & 104 & 106 & .. \\
\hline $\begin{array}{l}\text { Participants with no evidence of } \\
\text { disease }\end{array}$ & $48(40 \%)$ & $44(42 \%)$ & $50(47 \%)$ & $0 \cdot 48$ \\
\hline \multicolumn{5}{|l|}{ HIV negative at baseline } \\
\hline Participants followed up & 85 & 93 & 93 &.$\cdot$ \\
\hline $\begin{array}{l}\text { Participants with no evidence of } \\
\text { disease }\end{array}$ & $68(80 \%)$ & 77 (83\%) & $76(82 \%)$ & $0 \cdot 72$ \\
\hline \multicolumn{5}{|l|}{ HIV positive at baseline } \\
\hline Participants followed up & 109 & 95 & 101 & .. \\
\hline $\begin{array}{l}\text { Participants with no evidence of } \\
\text { disease }\end{array}$ & $50(46 \%)$ & $42(44 \%)$ & $55(54 \cdot 5)$ & $0 \cdot 36$ \\
\hline \multicolumn{5}{|c|}{$\begin{array}{l}\text { Data are } n \text {, or } n(\%) \text {. LLETZ=large loop excision of the transformation zone. HPV=human papillomavirus. VIA=visual } \\
\text { inspection with acetic acid. Treatment success was defined as either HPV type-specific clearance at } 6 \text { months among } \\
\text { women positive for the same HPV type at baseline, or negative VIA test at follow-up, if the baseline HPV test was } \\
\text { negative. *HPV reports were missing in six participants in the cryotherapy group, five participants in the thermal } \\
\text { ablation group, and five participants in the LLETZ group, who had HPV-positive results at baseline; these participants } \\
\text { were excluded from the analysis of treatment success proportion. }\end{array}$} \\
\hline
\end{tabular}

Table 2: Treatment success proportions at 6-months follow-up after treatment

interval between treatment and follow-up was 6.0 months (IQR 6.0-6.4 months) and the mean was 6.6 months (SD 1.8 months; range 4.8-19.6 months), with no difference between the treatment groups $(\mathrm{p}=0 \cdot 83)$.

Table 2 shows treatment success proportions at 6 months after treatment based on a combination of HPV test and VIA. HPV reports were missing in six participants in the cryotherapy group, five participants in the thermal ablation group, and five participants in the LLETZ group, and these individuals were excluded from the analysis. When based on HPV type-specific clearance and VIA-negative findings among participants who were HPV negative at baseline, the proportions of participants treated successfully were 120 (60\%) of 200 in the cryotherapy group, 123 (64\%) of 192 in the thermal ablation group, and 134 (67\%) of 199 in the LLETZ group $(p=0 \cdot 52)$. The proportions of participants with clearance of high-risk HPV at 6 months were similar between the cryotherapy (48 [40\%] of 121), thermal ablation (44 [42\%] of 104), and LLETZ (50 [47\%] of 106) groups $(\mathrm{p}=0 \cdot 48)$. The proportion of participants with clearance of HPV 16 (14 [43\%] of 32 in the cryotherapy group, 18 [64\%] of 28 in the thermal ablation group, and 18 [55\%] of 33 in the LLETZ group) was lower than the proportion of participants clearing HPV 18, HPV 45, or both (16 [100\%] of 16 in the cryotherapy group, 11 [69\%] of 16 in the thermal ablation group, and 14 [88\%] of 16 in the LLETZ group). Participants who were HIV-positive had lower treatment success than those who were HIV negative, irrespective of the treatment method (table 2). The proportions of successful treatment as reflected by VIA examination alone were similar across the groups (data not shown). The proportions of participants who had a normal VIA examination at follow-up were 162 (79\%) of 204 in the cryotherapy group, $162(84 \%)$ of 193 in the thermal ablation group, and $162(80 \%)$ of 203 in the LLETZ group $(\mathrm{p}=0 \cdot 47)$.

Almost all participants reported no or the least level of discomfort with their treatment, either immediately after or within 2 weeks of treatment, across all three groups (table 3). Few complained of moderate-tosevere pain immediately after the procedure and we found no statistically significant difference between the groups (table 3). When asked about the level of satisfaction with the service provided at the clinic and whether or not they would recommend the treatment to a friend, almost all women (99-100\%) in each of the three groups reported that they would, both immediately and at 2 weeks after treatment. Five deaths occured (three in the cryotherapy group and one each in the thermal ablation and LLETZ groups, due to intimate partner violence, suicide, metastatic breast cancer, renal failure of unknown cause, and complications following a excision of a soft tissue tumour on the thigh); none of the deaths were related to treatment. None of the participants reported any complications requiring medical consultation or hospitalisation.

$73(31 \%)$ of 238 participants who underwent LLETZ and had histological evidence of CIN 2-3 (table 4). More than half of the VIA-positive participants (124 [52\%] of 238) had some grade of CIN and none had invasive cancer. VIA-positive women aged 30-39 years had a higher proportion of CIN 2 or worse, compared with those aged 25-29 years and with those aged 40 years and older (table 4). In HIV-positive participants who were eligible for ablation, 55 (43\%) of 128 had CIN 2-3, compared with 17 (16\%) of 106 participants who were HIV negative. Of the HPV-positive (and VIA-positive) participants who had LLETZ, 64 (46\%) of 138 had histologically proven CIN 2-3, compared with only nine (9\%) of 100 who were HPV negative. Taking baseline combinations of high-risk HPV and HIV status, CIN 2-3 was detected in three $(5 \%)$ of 66 participants who were negative for HPV and HIV, six (19\%) of 32 participants who were HPV negative and HIV positive, 14 (35\%) of 40 participants who were HPV positive and HIV negative, and $49(51 \%)$ of 96 participants who were positive for HPV and HIV.

\section{Discussion}

To our knowledge, this study is the only report from a randomised study that assessed acceptability, safety, and performance of thermal ablation using a modern batterydriven portable machine. The most important finding of the preliminary report of our study is that thermal ablation appears to be acceptable to women and is associated with few side-effects. The early results of this 
trial suggest similar performance between thermal ablation and cryotherapy in terms of treatment success; however, this pilot study was not powered for this outcome.

The shorter treatment time, less cumbersome equipment, and non-dependance on refrigerant gas are distinct advantages of thermal ablation over cryotherapy. The cost of the battery driven thermal ablation is similar to that of standard cryotherapy equipment and a huge cost saving is expected because of low operational costs, if cryotherapy is replaced by thermal ablation. Moreover, current battery-operated thermal ablators are small, lightweight, and highly portable. Avoiding cumbersome gas tanks is a practical advantage to healthcare providers in LMICs.

Our results are particularly informative because of the high proportion of HIV-positive women in the study participants. The results suggest that thermal ablation using the Liger thermal ablator is safe in HIV-positive women. The preliminary results also suggest similar performance for the two ablative techniques in HIVpositive women, albeit substantially lower than that in HIV-negative women. Because the trial was implemented in routine health-care facilities in Zambia and treatment was provided by regular in-service nursing staff, the results could be generalisable to other LMIC settings.

Thermal ablation has been used extensively in Scotland since Semm first introduced the technique in Germany in the 1960 s, and more recently in many other parts of the world. In Scotland, Duncan ${ }^{16}$ has produced the largest and longest series of patients treated with thermal ablation (1453 patients followed up for $>14$ years) and the proportions of treatment success in his case series compare favourably with other treatment methods. ${ }^{17}$ The effectiveness of thermal ablation has been a ssessed in several meta-analyses. ${ }^{11,12}$ Randall and colleagues ${ }^{12}$ report an overall treatment success from 16 included studies for CIN2 or worse lesions of $93.6 \%$ (95\% CI $90 \cdot 8-96 \cdot 0)$. Treatment success was $92 \cdot 9 \%(90 \cdot 4-95 \cdot 1)$ for CIN1 or worse and was 89.0\% (84.0-95.0) for CIN 3. The only randomised controlled trial included in the analysis was done in Singapore. ${ }^{18}$ In that study, the authors reported no difference i $\mathrm{n} t$ reatment $\mathrm{s}$ uccess between cryotherapy and thermal ablation for any grades of CIN. The reported proportions of treatment success after thermal ablation in the Singapore study were $88.4 \%$ for CIN1, $84 \cdot 2 \%$ for CIN2, and $78 \cdot 6 \%$ for CIN3. Duan and colleagues ${ }^{19}$ have also presented the results of a randomised controlled trial of thermal ablation versus cryotherapy in a study of 149 women eligible for ablative therapy. In this study, thermal ablation was equallyor more effective than c ryotherapy w as a t $8 \mathrm{~m}$ onths, a $\mathrm{s}$ judged by HPV and cytological assessment.

There are several limitations in our study. The numbers of participants reported are small and the efficacy estimates should be interpreted with caution. The study continues to recruit eligible women and will do so until

\begin{tabular}{|c|c|c|c|c|}
\hline & $\begin{array}{l}\text { Cryotherapy } \\
\text { group }\end{array}$ & $\begin{array}{l}\text { Thermal ablation } \\
\text { group }\end{array}$ & LLETZ group & $\begin{array}{l}\text { Fisher's exact } \\
\text { p value }\end{array}$ \\
\hline \multicolumn{5}{|c|}{ Immediately after treatment } \\
\hline Participants assessed & 250 & 250 & 250 &. \\
\hline \multicolumn{5}{|c|}{ Intensity of pain or discomfort felt } \\
\hline 1 (no pain) & $120(48 \%)$ & $115(46 \%)$ & $134(54 \%)$ & 0.40 \\
\hline 2-3 (least pain) & $123(49 \%)$ & $129(52 \%)$ & $111(44 \%)$ & .. \\
\hline $4-6$ & $6(2 \%)$ & $3(1 \%)$ & $5(2 \%)$ & .. \\
\hline 7-9 (worst pain) & 0 & $1(<1 \%)$ & 0 & .. \\
\hline Unknown & $1(<1 \%)$ & $2(1 \%)$ & 0 & .. \\
\hline \multicolumn{5}{|c|}{ Level of satisfaction with the services } \\
\hline 1-3 (least satisfied) & 0 & 0 & 0 & 0.27 \\
\hline $4-6$ & $3(1 \%)$ & $1(<1 \%)$ & 0 &.. \\
\hline 7-9 (highly satisfied) & $246(98 \%)$ & $248(99 \%)$ & $250(100 \%)$ & .. \\
\hline Unknown & $1(<1 \%)$ & $1(<1 \%)$ & 0 &.$\cdot$ \\
\hline \multicolumn{5}{|c|}{ Will recommend the screening procedure to others } \\
\hline Yes & $248(99 \%)$ & $250(100 \%)$ & $249(100 \%)$ & 0.34 \\
\hline No & 0 & 0 & $1(<1 \%)$ &.$\cdot$ \\
\hline Cannot say & $2(<1 \%)$ & 0 & 0 & .. \\
\hline \multicolumn{5}{|l|}{2 weeks after treatment } \\
\hline Participants assessed & 241 & 242 & 237 & .. \\
\hline \multicolumn{5}{|c|}{ Intensity of pain or discomfort felt } \\
\hline 1 (no pain) & $214(89 \%)$ & $227(94 \%)$ & $208(88 \%)$ & 0.21 \\
\hline 2-3 (least pain) & $25(10 \%)$ & $15(6 \%)$ & $27(11 \%)$ &.$\cdot$ \\
\hline $4-6$ & $1(<1 \%)$ & 0 & $2(<1 \%)$ & .. \\
\hline 7-9 (worst pain) & 0 & 0 & 0 & .. \\
\hline \multicolumn{5}{|c|}{ Level of satisfaction with the services } \\
\hline 1-3 (least satisfied) & 0 & 0 & 0 & 0.55 \\
\hline $4-6$ & $1(<1 \%)$ & 0 & 0 &.. \\
\hline 7-9 (highly satisfied) & 239 (99\%) & $242(100 \%)$ & $237(100 \%)$ &. \\
\hline Unknown & $1(<1 \%)$ & 0 & 0 &.. \\
\hline \multicolumn{5}{|c|}{ Will recommend the screening procedure to others } \\
\hline Yes & $239(99 \%)$ & $242(100 \%)$ & $237(100 \%)$ & $0 \cdot 34$ \\
\hline No & 0 & 0 & 0 & $\cdot \cdot$ \\
\hline Cannot say & $2(<1 \%)$ & 0 & 0 &.$\cdot$ \\
\hline
\end{tabular}

Data are $n$, or $n(\%)$. LLETZ=large loop excision of the transformation zone.

Table 3: Intensity of pain and level of satisfaction reported immediately and 2 weeks after treatment

a sufficient sample size has been reached for the full trial. According to our sample size calculation, an additional 1000 participants need to be recruited in each group to give sufficient power to detect non-inferiority of thermal ablation as a treatment method for VIA-positive women compared with cryotherapy or LLETZ. A second caveat is that follow-up assessment at 6 months is probably too early to assess treatment success, but we do not anticipate any difference arising between the study groups, which are well balanced because of randomisation. Another limitation of the study is the absence of histopathology verification either at baseline or at followup. Ablative techniques are likely to be used widely in screen-and-treat settings and we have followed the standard of care (ie, VIA) to detect abnormalities before or after treatment. VIA is not a perfect screening test, with low-to-moderate sensitivity and low specificity. ${ }^{6}$ 


\begin{tabular}{|c|c|c|c|c|c|c|}
\hline & \multirow{2}{*}{$\begin{array}{l}\text { Participants with } \\
\text { histology report }\end{array}$} & \multicolumn{5}{|c|}{ Histological diagnosis at baseline } \\
\hline & & Normal & CIN1 & CIN2 & CIN3 & CIN2-3 \\
\hline Overall & $238 / 250(95 \%)$ & $114 / 238(48 \%)$ & $51 / 238(21 \%)$ & $32 / 238(13 \%)$ & $41 / 238(17 \%)$ & $73 / 238(31 \%)$ \\
\hline \multicolumn{7}{|l|}{ Age, years } \\
\hline $25-29$ & $81 / 84(96 \%)$ & $46 / 81(57 \%)$ & $20 / 81(25 \%)$ & $7 / 81(9 \%)$ & $8 / 81(10 \%)$ & $15 / 81(19 \%)$ \\
\hline 30-39 & $105 / 111(95 \%)$ & $42 / 105(40 \%)$ & $18 / 105(17 \%)$ & $21 / 105(20 \%)$ & $24 / 105(23 \%)$ & $45 / 105(43 \%)$ \\
\hline$\geq 40$ & $52 / 55(95 \%)$ & $26 / 52(50 \%)$ & $13 / 52(25 \%)$ & $4 / 52(8 \%)$ & $9 / 52(17 \%)$ & $13 / 52(25 \%)$ \\
\hline \multicolumn{7}{|l|}{ HPV test results } \\
\hline Negative & $100 / 105(95 \%)$ & $66 / 100(66 \%)$ & $25 / 100(25 \%)$ & $5 / 100(5 \%)$ & $4 / 100(4 \%)$ & $9 / 100(9 \%)$ \\
\hline Positive & $138 / 145(95 \%)$ & $48 / 138(35 \%)$ & $26 / 138(19 \%)$ & 27/138 (20\%) & $37 / 138(27 \%)$ & $64 / 138(46 \%)$ \\
\hline \multicolumn{7}{|l|}{ Baseline HIV status } \\
\hline Negative & $106 / 110(96 \%)$ & $60 / 106(57 \%)$ & 29/106 (27\%) & $7 / 106(7 \%)$ & $10 / 106(9 \%)$ & $17 / 106(16 \%)$ \\
\hline Positive & $128 / 135(95 \%)$ & $51 / 128(40 \%)$ & $22 / 128(17 \%)$ & 25/128 (20\%) & $30 / 128(23 \%)$ & $55 / 128(43 \%)$ \\
\hline \multicolumn{7}{|c|}{ Baseline HPV and HIV status combinations } \\
\hline HPV negative, HIV negative & $66 / 70(94 \%)$ & $44 / 66(67 \%)$ & $19 / 66(29 \%)$ & $2 / 66(3 \%)$ & $1 / 66(2 \%)$ & $3 / 66(5 \%)$ \\
\hline HPV negative, HIV positive & $32 / 33(97 \%)$ & $20 / 32(63 \%)$ & 6/32 (19\%) & $3 / 32(9 \%)$ & $3 / 32(9 \%)$ & 6/32 (19\%) \\
\hline HPV positive, HIV negative & $40 / 40(100 \%)$ & $16 / 40(40 \%)$ & $10 / 40(25 \%)$ & $5 / 40(13 \%)$ & $9 / 40(23 \%)$ & $14 / 40(35 \%)$ \\
\hline HPV positive, HIV positive & $96 / 102(94 \%)$ & $31 / 96(32 \%)$ & $16 / 96(17 \%)$ & $22 / 96(23 \%)$ & $27 / 96(28 \%)$ & $49 / 96(51 \%)$ \\
\hline
\end{tabular}

Table 4: Histology findings at baseline in the LLETZ group

However, WHO recommend VIA for LMICs that cannot afford an HPV detection test for population screening. The National Cancer Control Strategic Plan (2016-21) by the Zambian Ministry of Health also stipulates the use of VIA as the screening test of choice, followed by immediate cryotherapy or thermal ablation in all VIApositive women eligible for ablation. ${ }^{20}$ We implemented a pragmatic study and followed the existing protocol for VIA screen and treat. In the VIA screen-and-treat programme, a negative VIA result alone is considered as the test of cure. To increase the validity of our study, in addition to VIA, we used the most frequently used and powerful test of cure, which is a validated high-risk HPV test. This approach allowed us to investigate the association between HPV and VIA status and also to have a more valid endpoint than VIA alone. The results of HPV testing were masked because of management decisions. The low proportions of treatment success, as defined by HPV status, might reflect the early follow-up at 6 months and the possibility of recurrent infection, especially considering the high prevalence of HIV in participants. The follow-up VIA might have missed some of the lesions in HPV-negative participants because of the low sensitivity of the test. However, similar success proportions observed between the ablative and excisional treatment groups using the stringent criteria of disease clearance gives us confidence in the findings.

The duration of treatment with thermal ablation is still an unresolved issue. Randall and colleagues ${ }^{12}$ metaanalysis found that the proportion of patients achieving a cure did not vary significantly according to the duration of treatment. The proportions who were successfully treated were $92.9 \%$ in patients who were treated for $20 \mathrm{~s}$, $95.1 \%$ in those treated for $30 \mathrm{~s}$, and $84.8 \%$ in those treated for $45 \mathrm{~s}$. In Duncan' ${ }^{16}$ case series, $20 \mathrm{~s}$ applications were used but with the important caveat that where the probe tip did not cover the entire transformation zone, overlapping applications were made. ${ }^{16}$ The variables of probe tip size and the number of applications are, as yet, open questions. So far, in our study we used a duration of $40 \mathrm{~s}$ and multiple overlapping applications using a $20 \mathrm{~mm}$ probe. Our data and safety monitoring board has recommended that we reduce the treatment time to $30 \mathrm{~s}$ in the larger randomised controlled trial because the board members could not find evidence in the published literature for an advantage of a treatment duration of longer than $30 \mathrm{~s}$.

Reassuringly, very low frequencies of discomfort during and after treatment were reported in all three study groups. The frequencies reported in the procedure room during and immediately after treatment were slightly lower in the thermal ablation group, which might be explained by the shorter treatment duration associated with thermal ablation when compared with cryotherapy (45 s vs $11 \mathrm{~min}$ ). The low reporting of pain or cramps during LLETZ might be because local infiltration was routinely used for LLETZ but not for ablative treatment of either kind. Furthermore, similarly low discomfort was reported by participants after they had left the procedure room and by telephone 2 weeks after treatment.

All participants were advised to avoid penetrative sex for 6 weeks to reduce the risk of post-treatment bleeding and infection. Although an important concern for HIVinfected women, viral shedding does not increase after ablative treatment if women are already on antiretroviral 
therapy. ${ }^{21,22}$ All of our HIV-positive participants were on antiretroviral therapy at the time of VIA screening. Earlier studies have shown that $5-31 \%$ of women treated with cryotherapy did not comply with the advice for abstinence and the proportion of compliance increases with improved counseling. ${ }^{23}$ Although we ensured appropriate counseling of each participant, some particpants might have been non-compliant and therefore had bleeding or infection. However, we found that documenting the compliance proportion was difficult because of the cultural sensitivities around questions related to sexual practices.

One of the reasons that we included a third study group (excision by LLETZ) was to investgiate the histological diagnosis in VIA-positive women who were eligible for cryotherapy and thereby to better assess the test characteristics of visual inspection in its ability to discriminate between high-grade and low-grade or normal transformation zones. Thus, this approach allowed the analysis of the proportion of over-treatment specifically in the context of a screen-and-treat approach. Participants required a type 1 LLETZ, which has been shown to be safe in our study. Only $31 \%$ of women with a positive VIA and who were eligible for an ablative therapy had histologically proven high-grade CIN2 or worse. This positive predictive value (PPV) is higher for women in aged 30-39 years (43\%) and for those who are HIV positive (43\%). Much lower proportions (around 5\%) have been reported from studies that were done in regions with a low prevalence of cervical cancer. ${ }^{24} \mathrm{~A}$ metaanalysis of 26 cross-sectional studies by Sauvaget and colleagues observed that the average PPV of VIA to detect CIN2 or worse disease was $10 \% .^{6}$ The PPV in our study population was higher because of the high prevalence of HIV infection. Most of the earlier studies used punch biopsy as the gold standard to define disease status. Our study provides a more valid estimate of PPV by including the entire transformation zone in histopathological assessment. Nevertheless, the low PPV and resulting over-treatment (nearly half of the women treated had no CIN) in a VIA screen-and-treat programme is concerning. This finding also underscores the need for a safe treatment method that causes minimal discomfort to patients. We were reassured to know that no invasive cancer was inadvertently treated at least in the LLETZ group.

WHO has recently updated its clinical guidelines for the treatment of cervical precancer ${ }^{25}$ and now endorses the use of thermal ablation for ablative treatment. The low incidence of complications for either intervention reported by our study, especially in many HIV-positive women, formed part of the evidence for WHO to develop the new guidelines. A randomised controlled trial to compare thermal ablation with cryotherapy (using standard cryotherapy or cryopen) to treat histopathologically proved CIN2 or CIN3 is ongoing in Colombia, El Salvadore, and Peru (NCT03084081). If our early findings on the similar performance of the two ablative techniques are supported by our ongoing large trial and other studies, then health-care workers caring for screen-positive women in screen-and-treat programmes in LMICs could choose modern thermal ablation devices that are cordless, lightweight, and battery operated over cryotherapy instruments because of the practical disadvantages of cryotherapy. In view of the low incidence of histologically proven CIN2 or worse in VIAscreen positive women, the optimal screening test for LMICs should perhaps be reconsidered. We hope that less expensive HPV tests that do not require elaborate laboratory facilities will become available soon or alternative techniques-eg, artificial intelligence image recognition systems-will emerge.

\section{Contributors}

LFP implemented the study, oversaw data collection, analysed the data, drafted and revised the paper. GPP and PB designed the study protocols, oversaw the implementation of the study, analysed the data, and revised the draft manuscript. RM analysed and interpreted the data and revised the draft paper. EL, NN, CS, and MHM implemented the study and supported data collection, interpreted the data, and revised the draft paper. RS designed the protocol, interpreted the data, and revised the draft paper. WP designed the study, trained the study staff, interpreted the data, and prepared the draft paper.

\section{Declaration of interests}

WP has worked closely with Liger Medical to provide technical inputs in the design and development of the battery-operated thermal ablator during the past several years; he has not received any financial reward from the company. All other authors declare no competing interests.

\section{Data sharing}

External researchers can make written requests for sharing of data before publication or presentation. Requests will be assessed on a caseby-case basis in consultation with lead and co-investigators. A brief analysis plan and data request will be required and reviewed by the investigators for approval of data sharing. When requests are approved, data will be sent electronically in password protected files. All data sharing will abide by rules and policies defined by the sponsor; relevant institutional review boards; local, state, and federal laws and regulations. Data sharing mechanisms will ensure that the rights and privacy of individuals participating in research sponsored by the US National Institutes of Health will be protected at all times.

\section{Acknowledgments}

The study is fully funded by the US National Institute of Health (1UH2CA202721-01). LFP was funded by the University of North Carolina at Chapel Hill T32 Fellowship (5T32HD075731-05) and the University of Washington T32 Fellowship (5T32CA009515-34). The authors would like to give particular thanks to the women who participated in the study. The authors are grateful to Dean Wallace (LIGER/Cure Medical) for being the technical partner. The authors would also like to thank the following staff: Anne Njobvu, Besnart Ngandu, Susan Banda, Gift Kaputula, Beatrice Zulu, and Barbra Nakasote. The authors thank Krittika Guinot (IARC) for her help in the preparation of this manuscript. Where authors are identified as personnel of IARC or WHO, the authors alone are responsible for the views expressed in this article, which do not necessarily represent the decisions, policy, or views of IARC or WHO

\section{References}

1 Wilson JMG, Jungner G. Principles and practice of screening for disease. Geneva: WHO, 1968.

2 Prendiville W, Denny L. Cancer of the cervix: early detection and cost-effective solutions. Int J Gynaecol Obstet 2015; 131: S28-32.

3 Prendiville W, Cullimore J, Norman S. Large loop excision of the transformation zone (LLETZ). A new method of management for women with cervical intraepithelial neoplasia. Br J Obstet Gynaecol 1989; 96: 1054-60. 
4 Prendiville W, Sankaranarayanan R. Colposcopy and treatment of cervical precancer. IARC technical publication No 45. Lyon: IARC 2017.

5 Semm K. New apparatus for the "cold-coagulation" of benign cervical lesions. Am J Obstet Gynecol. 1966; 95: 963-66.

6 Sauvaget C, Fayette JM, Muwonge R, Wesley R, Sankaranarayanan R. Accuracy of visual inspection with acetic acid for cervical cancer screening. Int J Gynaecol Obstet 2011; 113: 14-24.

7 Basu P, Meheus F, Chami Y, Hariprasad R, Zhao F,

Sankaranarayanan R. Management algorithms for cervical cancer screening and precancer treatment for resource-limited settings. Int J Gynaecol Obstet 2017; 138: 26-32.

8 WHO. Guidelines for screening and treatment of precancerous lesions for cervical cancer prevention. Geneva: WHO, 2013.

9 Basu P, Mittal S, Bhadra Vale D, Chami Kharaji Y. Secondary prevention of cervical cancer. Best Pract Res Clin Obstet Gynaecol 2018; 47: 73-85.

10 Maza M, Schocken CM, Bergman KL, Randall TC, Cremer ML. Cervical precancer treatment in low- and middle-income countries: a technology overview. J Glob Oncol 2016; 3: 400-08.

11 Dolman L, Sauvaget C, Muwonge R, Sankaranarayanan R. Meta-analysis of the efficacy of cold coagulation as a treatment method for cervical intraepithelial neoplasia: a systematic review. BJOG 2014; 121: 929-42.

12 Randall TC, Sauvaget C, Muwonge R, Trimble EL, Jeronimo J. Worthy of further consideration: an updated meta-analysis to address the feasibility, acceptability, safety and efficacy of thermal ablation in the treatment of cervical cancer precursor lesions. Prev Med 2019; 118: 81-91.

13 Sankaranarayanan R, Wesley R. A practical manual on visua screening for cervical neoplasia. IARC Technical Publication No. 41 Lyon: IARC, 2003.

14 Bornstein J, Bentley J, Bösze P, et al. 2011 colposcopic terminology of the International Federation for Cervical Pathology and Colposcopy. Obstet Gynecol 2012; 120: 166-72.

15 Basu P, Sankaranarayanan R (2017). Atlas of colposcopy-principles and practice: IARC CancerBase No. 13. Lyon, France: IARC, 2017. https://screening.iarc.fr/atlascolpo.php (accessed Aug 26, 2019).
16 Duncan ID. The Semm cold coagulator in the management of cervical intraepithelial neoplasia. Clin Obstet Gynecol 1983; 126: 996-1006.

17 Gordon HK, Duncan ID. Effective destruction of cervical intraepithelial neoplasia (CIN) 3 at 100 degrees C using the Semm cold coagulator: 14 years experience. Br J Obstet Gynaecol 1991; 98: $14-20$

18 Singh P, Loke K-L, Hii JHC, et al. Cold coagulation versus cryotherapy for treatment of cervical intraepithelial neoplasia: results of a prospective randomized trial.

Colposcopy Gynecol Laser Surg 1988; 4: 211-21.

19 Duan L, Du H, Liu A, et al. Thermo-ablation vs cryo for the treatment of cervical pre-cancers. ASCCP 2019 Annual Scientific Meeting on Anogenital and HPV-Related Diseases; Atlanta GA, USA; April 4-7, 2019.

20 Ministry of Health, Republic of Zambia. National cancer control strategic plan 2016-21. Lusaka, Zambia: Ministry of Health. https://www.iccp-portal.org/system/files/plans/NCCSP\%20 Final\%20Version\%20Zambia.pdf (accessed Aug 26, 2019).

21 Chung MH, McKenzie KP, Richardson BA, et al. Cervical HIV-1 RNA shedding after cryotherapy among HIV-positive women with cervical intraepithelial neoplasia stage 2 or 3. AIDS 2011; 25: 1915-19.

22 Franceschi S. Past and future of prophylactic ablation of the cervical squamocolumnar junction. Ecancermedicalscience 2015; 9: 527

23 Jacob M, Broekhuizen FF, Castro W, Sellors J. Experience using cryotherapy for treatment of cervical precancerous lesions in low-resource settings. Int J Gynaecol Obstet 2005; 89: S13-20.

24 Basu P, Banerjee D, Mittal S, et al. Evaluation of a compact, rechargeable, magnifying device to triage VIA and HPV positive women in a cervical cancer screening program in rural India. Cancer Causes Control 2016; 27: 1253-59.

25 WHO. WHO guidelines for the use of thermal ablation for cervical pre-cancer lesions. Geneva: World Health Organization, 2019. https://apps.who.int/iris/bitstream/handle/10665/329299/ 9789241550598-eng.pdf? sequence=1\&isAllowed=y (accessed Oct 30, 2019). 